\title{
A GENERALIZATION OF THE CLASSICAL PARTITION THEOREMS
}

\author{
BY
}

\author{
GEORGE E. ANDREWS( $\left.{ }^{1}\right)$
}

1. Introduction. Let $P_{d}(n)$ denote the number of partitions of $n$ into positive integers of the form $n=b_{1}+\cdots+b_{s}$ with $b_{i}-b_{i+1} \geqq d$ where strict inequality holds if $d \mid b_{i} . P_{1}(n)$ appears in the first Rogers-Ramanujan identity [5, p. 291]. $P_{2}(n)$ appears in the first of the Göllnitz-Gordon partition theorems [2, p. 945], and $P_{3}(n)$ appears in a partition theorem of I. J. Schur [6, p. 489].

For $d>3$, we have the following result due to H. L. Alder [1, p. 713].

(1.1) Let $S$ be any fixed set of positive integers. Then $P_{d}(n)$ is not identically equal to the number of partitions of $n$ into parts taken from $S$ if $d>3$.

In a different approach, B. Gordon [4, p. 394] has made the following generalization of the Rogers-Ramanujan identities.

(1.2) Let $a$ and $k$ be integers with $0<a \leqq k$. Let $A_{k, a}(n)$ denote the number of partitions of $n$ into parts $\not \equiv 0, \pm a(\bmod 2 k+1)$. Let $B_{k, a}(n)$ denote the number of partitions of $n$ of the form $n=b_{1}+\cdots+b_{s}$ with $b_{i} \geqq b_{i+1}, b_{i}-b_{i+k-1} \geqq 2$, and with 1 appearing as a summand at most $a-1$ times. Then $A_{k, a}(n)=B_{k, a}(n)$.

The Göllnitz-Gordon identities have also been generalized in this manner [2, p. 946].

(1.3) Let $a$ and $k$ be integers with $0<a \leqq k$. Let $A_{k, a}^{\#}(n)$ denote the number of partitions of $n$ into parts which are neither $\equiv 2(\bmod 4)$ nor $\equiv 0, \pm(2 a-1)(\bmod 4 k)$. Let $B_{k, a}^{\#}(n)$ denote the number of partitions of $n$ of the form $n=b_{1}+\cdots+b_{s}$, with $b_{i} \geqq b_{i+1}$, no odd parts are repeated, $b_{i}-b_{i+k-1} \geqq 2$ with strict inequality if $2 \mid b_{i}$ and at most $a-1$ parts not exceeding 2 appear. Then $A_{k, a}^{\#}(n)=B_{k, a}^{\#}(n)$.

This leads us to define the following partition function. Let $B_{\lambda, k, a}(n)$ denote the number of partitions of $n$ of the form $n=b_{1}+\cdots+b_{s}$, with $b_{i} \geqq b_{i+1}$, no parts $\not \equiv 0(\bmod \lambda+1)$ are repeated, $b_{i}-b_{i+k-1} \geqq \lambda+1$ with strict inequality if $\lambda+1 \mid b_{i}$ and at most $a-1$ parts not exceeding $\lambda+1$ appear. We note that $B_{0, k, a}(n)$ appears in (1.2); $B_{1, k, a}(n)$ appears in (1.3), and $B_{d-1,2,2}(n)=P_{d}(n)$.

QUESTION. If $k$ is sufficiently large with respect to $\lambda$, does $B_{\lambda, k, a}(n)$ appear in $a$ partition theorem of the Rogers-Ramanujan type?

We answer this question affirmatively with the following result.

THEOREM 1. Let $0 \leqq \lambda \leqq a \leqq k$ be integers, and $2 \lambda-1 \leqq k$. If $\lambda$ is even, define

Received by the editors December 6, 1967.

(1) Partially supported by National Science Foundation Grant GP-6663.

Copyright (C) 1969, American Mathematical Society 
$A_{\lambda, k, a}(n)$ to be the number of partitions of $n$ into parts such that no part $\not \equiv 0(\bmod \lambda+1)$ may be repeated and no part is $\equiv 0, \pm\left(a-\frac{1}{2} \lambda\right)(\lambda+1)(\bmod (2 k-\lambda+1)(\lambda+1))$. If $\lambda$ is odd, define $A_{\lambda, k, a}(n)$ to be the number of partitions of $n$ into parts such that no part $\not \equiv 0\left(\bmod \frac{1}{2}(\lambda+1)\right)$ may be repeated, no part is $\equiv \lambda+1(\bmod 2 \lambda+2)$, and no part is $\equiv 0, \pm(2 a-\lambda) \frac{1}{2}(\lambda+1)(\bmod (2 k-\lambda+1)(\lambda+1))$. Then $A_{\lambda, k, a}(n)=B_{\lambda, k, a}(n)$.

There is numerical evidence to suggest that the condition " $k \geqq 2 \lambda-1$ " is unnecessary; but, the proof given here strongly relies on this condition. Actually the case $k=\lambda=a=2$ violates this condition; however, Theorem 1 becomes Schur's theorem in this case.

In $\S 2$, we shall develop results concerning auxiliary partition functions. In $\S 3$, we shall prove Theorem 1; our work will be based on the generalized well-poised basic hypergeometric functions studied in [3]. In $\$ 4$, we prove a more general theorem than Theorem 1 in which the parameter $a$ is allowed to take values in the interval $\left(\frac{1}{2} \lambda, k\right]$. In $\S 5$, we discuss possibilities for further work.

2. Auxiliary partition functions. Let $\pi(k, \lambda, t, j, \mu ; n)$ denote the number of partitions of $n$ of the form $n=\sum_{i=1}^{2 \lambda+1} f_{i} \cdot i$ (here $f_{i}$ is the number of times the summand $i$ appears) where

(2.1) $f_{1}+\cdots+f_{\lambda+1}=k-\lambda+j$;

(2.2) $f_{\lambda+1}+\cdots+f_{2 \lambda+1}=k-t$;

(2.3) $f_{m}+\cdots+f_{m+\lambda} \geqq k$ for some $m$;

(2.4) $f_{a}>1$ implies $a=\lambda+1$;

(2.5) $f_{1}+\cdots+f_{2 \lambda+1}=k+\mu$.

Throughout our discussion we shall assume $k, \lambda, t, j$, and $\mu$ are all nonnegative integers with $j \leqq \lambda, k \geqq \max (1,2 \lambda-1)$, and $\pi(k, \lambda, t, j, \mu ; 0)=0$.

Let $g(k, \lambda, t, j, \mu ; q)=\sum_{n=0}^{\infty} \pi(k, \lambda, t, j, \mu ; n) q^{n}$.

\section{LEMMA 2.1.}

$$
g(k, \lambda, t, j, \mu ; q)=q^{(k-t)(\lambda+1)} \sigma_{\mu}(\lambda) \sigma_{\lambda+\mu+t-j}(\lambda),
$$

where

$$
\begin{array}{rlrl}
\sigma_{i}(\lambda) & =q^{i(i+1) / 2}\left[\begin{array}{l}
\lambda \\
i
\end{array}\right], & \\
& =q^{i(i+1) / 2} \frac{\left(q^{\lambda}-1\right) \cdots\left(q^{\lambda-i+1}-1\right)}{\left(q^{i}-1\right) \cdots(q-1)}, & & 0 \leqq i \leqq \lambda, \\
& =0, & & \text { otherwise }
\end{array}
$$

thus $\sigma_{i}(\lambda)$ is just the generating function for partitions into exactly $i$ distinct parts with all parts $\leqq \lambda$.

Proof. We proceed by induction on the second argument of $g$, namely $\lambda$. If $\lambda=0$, then since $j \leqq \lambda, j=0$. The conditions (2.1)-(2.5) imply $f_{1}=k+j=k-t$ 
$=k+\mu \geqq k$; thus $j=t=\mu=0$. Thus the only allowable partition is of $k$ into $k$ "ones". Therefore

$$
\begin{aligned}
g(k, 0, t, j, \mu ; q) & =q^{k} & & \text { if } \mu=j=t=0 \\
& =0 & & \text { otherwise. }
\end{aligned}
$$

On the other hand, consider the right-hand side of (2.6); call it $G(k, 0, t, j, \mu ; q)$. The only way $G(k, 0, t, j, \mu ; q)$ will be nonzero is if $\mu=0, t=j$, and since $j \leqq \lambda$ we have $t=j=0$. Hence

$$
\begin{aligned}
G(k, 0, t, j, \mu ; q) & =q^{k} & & \text { if } \mu=j=t=0 \\
& =0 & & \text { otherwise. }
\end{aligned}
$$

Thus the lemma holds for $\lambda=0$.

If $\mu=0$, there are just $k$ parts appearing in the partition by (2.5), and since (2.3) must hold we see that there exists a particular $m$ such that

$$
f_{m}+\cdots+f_{m+\lambda}=k, \quad f_{i}=0 \text { for } i<m \text { or } i>m+\lambda .
$$

If we set $A=f_{1}+\cdots+f_{\lambda}, B=f_{\lambda+2}+\cdots+f_{2 \lambda+1}$, then (2.1), (2.2), and (2.5) imply $A=t+\mu, f_{\lambda+1}=k+j-\lambda-t-\mu, B=\lambda+\mu-j$; thus in case $\mu=0, A=t, f_{\lambda+1}=$ $k+j-\lambda-t, B=\lambda-j$. Let us now consider any partition of the type described in this paragraph. If we subtract $\lambda+1$ from each of the $\lambda-j$ parts greater than $\lambda+1$, we get a partition with $\lambda+1$ appearing $k+j-\lambda-t$ times with $\lambda+t-j$ remaining parts which are distinct and are all $\leqq \lambda$. Conversely if we have a given partition in which $\lambda+1$ appears $k+j-\lambda-t$ times and there are $\lambda+t-j$ remaining parts which are distinct and are $\leqq \lambda$, we define $m$ to be the integer such that $f_{1}+\cdots+f_{m-1}=\lambda-j$, and we add $\lambda+1$ to each part $<m$; this gives us precisely the type of partition described at the beginning of this paragraph. Hence by the definition of $\sigma_{j}(\lambda)$,

$$
\begin{aligned}
g(k, \lambda, t, j, 0 ; q) & =q^{(k+j-\lambda-t)(\lambda+1)+(\lambda-j)(\lambda+1)} \sigma_{\lambda+t-j}(\lambda) \\
& =q^{(k-t)(\lambda+1)} \sigma_{\lambda+t-j}(\lambda) \\
& =G(k, \lambda, t, j, 0 ; q)
\end{aligned}
$$

and (2.6) is verified in this case.

We now proceed by induction on $\lambda(>0)$, and we may also assume $\mu>0$. Our first task is to verify

$$
\begin{aligned}
\pi(k, \lambda, t, j, \mu ; n)= & \pi(k, \lambda-1, t, j-1, \mu ; n+j-k-2 \mu-\lambda) \\
& +\pi(k, \lambda-1, t, j, \mu ; n+j-k-2 \mu-\lambda) \\
& +\pi(k, \lambda-1, t, j-2, \mu-1 ; n+j-k-2 \mu-\lambda) \\
& +\pi(k, \lambda-1, t, j-1, \mu-1 ; n+j-k-2 \mu-\lambda) .
\end{aligned}
$$

To verify (2.7) we proceed as follows. Let us consider a partition of $n$ of the type enumerated by $\pi(k, \lambda, t, j, \mu ; n)$. Let us subtract 1 from every summand $\leqq \lambda+1$, and let us subtract 2 from each summand $>\lambda+1$. The number being partitioned now is $n-(k+j-\lambda)-2(\lambda+\mu-j)=n+j-k-2 \mu-\lambda$. Four cases are to be 
considered depending on: (1) $f_{1}=f_{\lambda+2}=0$; (2) $f_{1}=0, f_{\lambda+2}=1$; (3) $f_{1}=1, f_{\lambda+2}=0$; (4) $f_{1}=f_{\lambda+2}=1$.

We let $f_{i}^{\prime}$ denote the number of appearances of $i$ in the transformed partition. Thus for example in case (1), conditions (2.1)-(2.5) now read

$(2.1)_{1} f_{1}^{\prime}+\cdots+f_{\lambda}^{\prime}=k-(\lambda-1)+j-1$;

(2.2) $f_{\lambda}^{\prime}+\cdots+f_{2 \lambda-1}^{\prime}=k-t$;

(2.3) ${ }_{1} f_{m}^{\prime}+\cdots+f_{m+\lambda-1}^{\prime} \geqq k$ for some $m$;

(2.4) $f_{a}^{\prime}>1$ implies $a=\lambda$;

$(2.5)_{1} f_{1}^{\prime}+\cdots+f_{2 \lambda-1}^{\prime}=k+\mu$.

Thus we have a partition of the type enumerated by

$$
\pi(k, \lambda-1, t, j-1, \mu ; n+j-k-2 \mu-\lambda) .
$$

Indeed the above procedure establishes a one-to-one correspondence between those partitions enumerated by $\pi(k, \lambda, t, j, \mu ; n)$ which fall in case (1) and the partitions enumerated by $\pi(k, \lambda-1, t, j-1, \mu ; n+j-k-2 \mu-\lambda)$. Similarly in the other three cases we get the other three partition functions appearing on the righthand side of equation (2.7). Thus (2.7) is established.

(2.7) implies

$$
\begin{aligned}
g(k, \lambda, t, j, \mu ; q)=q^{k+\lambda+2 \mu-j}(g(k, \lambda-1, t, j-1 & , \mu ; q)+g(k, \lambda-1, t, j, \mu ; q) \\
& +g(k, \lambda-1, t, j-2, \mu-1 ; q) \\
& +g(k, \lambda-1, t, j-1, \mu-1 ; q))
\end{aligned}
$$

Let us now examine the right-hand side of (2.6) which we have called $G(k, \lambda, t, j, \mu ; q)$. Now

$$
\sigma_{j}(\lambda)=q^{j} \sigma_{j}(\lambda-1)+q^{j} \sigma_{j-1}(\lambda-1) ;
$$

this is merely a restatement of the well-known formula for Gaussian polynomials

$$
\left[\begin{array}{l}
\lambda \\
j
\end{array}\right]=\left[\begin{array}{c}
\lambda-1 \\
j-1
\end{array}\right]+q^{j}\left[\begin{array}{c}
\lambda-1 \\
j
\end{array}\right]
$$

Consequently

$$
\begin{aligned}
G(k, \lambda, t, j, \mu ; q)= & q^{(k-t)(\lambda+1)} \sigma_{\mu}(\lambda) \sigma_{\lambda+\mu+t-j}(\lambda) \\
= & q^{k-t+\mu+\lambda+\mu+t-j} q^{(k-t) \lambda}\left(\sigma_{\mu}(\lambda-1)+\sigma_{\mu-1}(\lambda-1)\right) \\
& \quad \times\left(\sigma_{\lambda+\mu+t-j}(\lambda-1)+\sigma_{\lambda+\mu+t-j-1}(\lambda-1)\right) \\
= & q^{k+\lambda+2 \mu-j}(G(k, \lambda-1, t, j-1, \mu ; q)+G(k, \lambda-1, t, j, \mu ; q) \\
& \quad+G(k, \lambda-1, t, j-2, \mu-1 ; q) \\
& \quad+G(k, \lambda-1, t, j-1, \mu-1 ; q)) .
\end{aligned}
$$

Now we know that $g(k, \lambda, t, j, \mu ; q)$ and $G(k, \lambda, t, j, \mu ; q)$ are identical if either $\lambda$ or $\mu$ is zero. Thus since they both satisfy the recurrence (2.8), we have by induction on $\lambda$ that they are equal in general. Thus Lemma 2.1 is established. 
Lemma 2.2. Let $\pi_{1}(k, \lambda, t, j ; m ; n)$ denote the number of partitions of $n$ into $m$ parts of the form $n=\sum_{i=1}^{2 \lambda+1} f_{i} \cdot i$, where

(2.12) $f_{1}+\cdots+f_{\lambda+1} \leqq k-\lambda+j$;

(2.13) $f_{\lambda+1}+\cdots+f_{2 \lambda+1}=k-t$;

(2.14) $f_{v}+\cdots+f_{v+\lambda} \geqq k$ for some $v$;

(2.15) $f_{a}>1$ implies $a=\lambda+1$.

Let

Then

$$
g_{1}(k, \lambda, t, j ; x ; q)=\sum_{m=1}^{\infty} \sum_{n=1}^{\infty} x^{m} q^{n} \pi_{1}(k, \lambda, t, j ; m ; n)
$$

$$
g_{1}(k, \lambda, t, j ; x ; q)=x^{k} q^{(k-t)(\lambda+1)} \sum_{\mu=0}^{\lambda} x^{\mu} \sigma_{\mu}(\lambda) \sum_{r=0}^{j-\mu-t} \sigma_{\lambda-r}(\lambda) .
$$

Proof. By the definitions of $\pi$ and $\pi_{1}$, it is clear that

Hence

$$
\pi_{1}(k, \lambda, t, j ; k+\mu ; n)-\pi_{1}(k, \lambda, t, j-1 ; k+\mu ; n)=\pi(k, \lambda, t, j, \mu ; n) .
$$

$$
\begin{aligned}
g_{1}(k, \lambda, t, j ; x ; q)-g_{1}(k, \lambda, t, j-1 ; x ; q) & =\sum_{\mu=0}^{\infty} x^{k+\mu} g(k, \lambda, t, j, \mu ; q) \\
& =\sum_{\mu=0}^{\infty} x^{k+\mu} q^{(k-t)(\lambda+1)} \sigma_{\mu}(\lambda) \sigma_{\lambda+\mu+t-j}(\lambda) .
\end{aligned}
$$

Therefore

$$
\begin{aligned}
g_{1}(k, \lambda, t, j ; x ; q) & =\sum_{r=\lambda-k}^{j} \sum_{\mu=0}^{\infty} x^{k+\mu} q^{(k-t)(\lambda+1)} \sigma_{\mu}(\lambda) \sigma_{\lambda+\mu+t-r}(\lambda) \\
& =x^{k} q^{(k-t)(\lambda+1)} \sum_{\mu=0}^{\lambda} x^{\mu} \sigma_{\mu}(\lambda) \sum_{r=\mu+t}^{j} \sigma_{\lambda+\mu+t-r}(\lambda) \\
& =x^{k} q^{(k-t)(\lambda+1)} \sum_{\mu=0}^{\lambda} x^{\mu} \sigma_{\mu}(\lambda) \sum_{r=0}^{j-\mu-t} \sigma_{\lambda-r}(\lambda) .
\end{aligned}
$$

To conclude this section we prove a result concerning the multiplication of generating functions.

Lemma 2.3. Let $p_{1}(A ; c ; m, n)$ denote the number of partitions of $n$ into $m$ parts all $<c$ where the summands are subject to a set of conditions, $A$. Let $p_{2}(B ; c ; m, n)$ denote the number of partitions of $n$ into $m$ parts all $\geqq c$ where the summands are subject to a set of conditions, $B$. Let

$$
\begin{aligned}
& f_{1}(x, q)=\sum_{m=0}^{\infty} \sum_{n=0}^{\infty} p_{1}(A ; c ; m, n) x^{m} q^{n}, \\
& f_{2}(x, q)=\sum_{m=0}^{\infty} \sum_{n=0}^{\infty} p_{2}(B ; c ; m, n) x^{m} q^{n}
\end{aligned}
$$

where we assume that $f_{i}(x, q)$ is absolutely convergent for $|x|<b_{i},|q|<1$. 
Then for $|x|<\min \left(b_{1}, b_{2}\right),|q|<1$, if we let

$$
f_{1}(x, q) f_{2}(x, q)=\sum_{m=0}^{\infty} \sum_{n=0}^{\infty} p_{3}(A, B ; c ; m, n) x^{m} q^{n},
$$

then $p_{3}(A, B ; c ; m, n)$ is the number of partitions of $n$ into $m$ parts such that the summands $<c$ are subject to condition $A$ and the summands $\geqq c$ are subject to condition $B$.

Proof The result follows directly from

$$
p_{3}(A, B ; c ; m, n)=\sum_{m_{1}+m_{2}=m_{m} n_{1}+n_{2}=n} p_{1}\left(A ; c ; m_{1}, n_{1}\right) p_{2}\left(B ; c ; m_{2}, n_{2}\right) .
$$

3. Proof of Theorem 1. We begin by studing certain $q$-series considered in [3]. As in $\S 2$, we require that $k \geqq \max (1,2 \lambda-1)$. We define

$$
\begin{aligned}
& J_{\lambda, k, i}(x)=J_{\lambda, k, i}(x ; q)=J_{k, i}\left(-q,-q^{2}, \ldots,-q^{\lambda} ; x ; q^{\lambda+1}\right) ; \\
& H_{\lambda, k, i}(x)=H_{\lambda, k, i}(x ; q)=H_{k, i}\left(-q,-q^{2}, \ldots,-q^{\lambda} ; x q^{\lambda+1} ; q^{\lambda+1}\right),
\end{aligned}
$$

where (as in [3])

$$
\begin{aligned}
J_{k, i}\left(a_{1}, \ldots, a_{\lambda} ; x ; q\right) & \\
= & \sum_{n=0}^{\infty}(-1)^{n(\lambda+1)} x^{k n}\left(a_{1} a_{2} \cdots a_{\lambda}\right)^{-n} q^{(2 k-\lambda+1) n^{2} / 2+(\lambda+1) n / 2+(k-i) n} \\
& \cdot \prod_{r=1}^{n}\left(1-q^{r}\right)^{-1} \prod_{u=n+1}^{\infty}\left(1-x q^{u}\right)^{-1} \prod_{s=1}^{\lambda}\left(\prod_{v=0}^{n-1}\left(1-a_{s} q^{v}\right) \prod_{w=n+1}^{\infty}\left(1-\frac{x q^{w}}{a_{s}}\right)\right) \\
& \cdot\left\{1+\frac{(-1)^{\lambda+1} x^{i} q^{(2 n+1) i-\lambda n}\left(1-a_{1} q^{n}\right)\left(1-a_{2} q^{n}\right) \cdots\left(1-a_{\lambda} q^{n}\right)}{a_{1} a_{2} \cdots a_{\lambda}\left(1-x q^{n+1} a_{1}^{-1}\right)\left(1-x q^{n+1} a_{2}^{-1}\right) \cdots\left(1-x q^{n+1} a_{\lambda}^{-1}\right)}\right\} \\
H_{k, i}\left(a_{1}, \ldots, a_{\lambda} ; x ; q\right) & \\
= & \sum_{n=0}^{\infty}(-1)^{n(\lambda+1)} x^{k n}\left(a_{1} a_{2} \cdots a_{\lambda}\right)^{-n} q^{(2 k-\lambda+1) n^{2} / 2+(\lambda+1) n / 2-i n}\left(1-x^{i} q^{2 n i}\right) \\
& \cdot \prod_{r=1}^{n}\left(1-q^{r}\right)^{-1} \prod_{u=n}^{\infty}\left(1-x q^{u}\right)^{-1} \prod_{s=1}^{\lambda}\left(\prod_{v=0}^{n-1}\left(1-a_{s} q^{v}\right) \prod_{w=n+1}^{\infty}\left(1-\frac{x q^{w}}{a_{s}}\right)\right) .
\end{aligned}
$$

Then from [3, equation (2.1)], we have

$$
H_{\lambda, k, i}(x)-H_{\lambda, k, i-1}(x)=\left(x q^{\lambda+1}\right)^{i-1} J_{\lambda, k, k-1+1}\left(x q^{\lambda+1}\right) ;
$$

from [3, equation (2.2)], we have

$$
J_{\lambda, k, i}(x)=\sum_{j=0}^{\lambda} x^{j} \sigma_{j}(\lambda) H_{\lambda, k, i-j}(x) ;
$$

from [3, equation (2.3)], we have

$$
H_{\lambda, k,-i}(x)=-\left(x q^{\lambda+1}\right)^{-i} H_{\lambda, k, i}(x),
$$


and from [3, equation (2.8)], we have

$$
H_{\lambda, k, 1}(x)=J_{\lambda, k, k}\left(x q^{\lambda+1}\right)=J_{\lambda, k, k+1}\left(x q^{\lambda+1}\right) .
$$

LemmA 3.1. Let $|q|<1$, and let $H_{\lambda, k, i}^{*}(x)(0 \leqq i \leqq k)$ and $J_{\lambda, k, i}^{*}(x)(1 \leqq i \leqq k)$ be any collection of functions analytic in $x$ in the neighborhood of zero which satisfy

$$
\begin{gathered}
H_{\lambda, k, i}^{*}(x)-H_{\lambda, k, i-1}^{*}(x)=\left(x q^{\lambda+1}\right)^{i-1} J_{\lambda, k, k-i+1}^{*}\left(x q^{\lambda+1}\right), \quad 1 \leqq i \leqq k ; \\
H_{\lambda, k, 0}^{*}(x)=0 ; \\
J_{\lambda, k, i}^{*}(x)=\sum_{j=0}^{\min (i, \lambda)} x^{j} \sigma_{j}(\lambda) H_{\lambda, k, i-j}^{*}(x) \\
-x^{i} \sum_{j=\min (i, \lambda)+1}^{\lambda} q^{(\lambda+1)(i-j)} \sigma_{j}(\lambda) H_{\lambda, k, j-i}^{*}(x) ; \\
H_{\lambda, k, i}^{*}(0)=J_{\lambda, k, i}^{*}(0)=1, \quad 1 \leqq i \leqq k .
\end{gathered}
$$

Then $H_{\lambda, k, i}^{*}(x)=H_{\lambda, k, i}(x)$, and $J_{\lambda, k, i}^{*}(x)=J_{\lambda, k, i}(x)$.

Proof. We need only show that solutions of the above equations are unique since (3.3)-(3.8) imply that the $H_{\lambda, k, i}(x)$ and $J_{\lambda, k, i}(x)$ fulfill the conditions of the lemma. If we can show that the $H_{\lambda, k, i}^{*}(x)$ are uniquely determined, then since the $J_{\lambda, k, i}^{*}(x)$ are defined in terms of the $H_{\lambda, k, i}^{*}(x)$ by (3.11), the $J_{\lambda, k, i}^{*}(x)$ will be uniquely determined also.

From (3.9) and (3.11) we have

$$
\begin{aligned}
& H_{\lambda, k, 2}^{*}(x)-H_{\lambda, k, i-1}^{*}(x) \\
& =\left(x q^{\lambda+1}\right)^{i-1} \sum_{j=0}^{\min (k-i+1, \lambda)} x^{j} q^{j(\lambda+1)} \sigma_{j}(\lambda) H_{\lambda, k, k-i+1-j}^{*}\left(x q^{\lambda+1}\right) \\
& \quad-\left(x q^{\lambda+1}\right)^{k-i+1} \sum_{j=\min (k-i+1, \lambda)+1}^{\lambda} q^{(\lambda+1)(k-i+1-j)} \sigma_{j}(\lambda) H_{\lambda, k, j-k+i-1}^{*}\left(x q^{\lambda+1}\right) .
\end{aligned}
$$

Hence if $H_{\lambda, k, i}^{*}(x)=\sum_{n=0}^{\infty} \eta_{n}(i) x^{n}$, then by (3.10) and (3.12)

$$
\begin{aligned}
\eta_{0}(i) & =1, \quad 1 \leqq i \leqq k, \\
& =0, \quad i=0 .
\end{aligned}
$$

From (3.13),

$$
\begin{aligned}
\eta_{n}(1)-\eta_{n}(k) q^{n(\lambda+1)} & =\text { polynomial in } \eta_{j} \text { 's with } j<n, \\
\eta_{n}(2)-\eta_{n}(1) & =\text { polynomial in } \eta_{j} \text { 's with } j<n, \\
& \vdots \\
\eta_{n}(k)-\eta_{n}(k-1) & =\text { polynomial in } \eta_{j} \text { 's with } j<n .
\end{aligned}
$$


We consider the above equations as a system of $k$ equations in the $k$ unknowns $\eta_{n}(1), \eta_{n}(2), \ldots, \eta_{n}(k)$. The determinant of the system is $1-q^{n(\lambda+1)} \neq 0$ since $|q|<1$. Hence the $\eta_{n}(i)$ are uniquely determined by the $\eta_{j}$ 's with $j<n$. Hence by mathematical induction the $\eta_{n}(i)$ are unique, and therefore the $H_{\lambda, k, i}^{*}(x)$ are unique. Thus Lemma 3.1 is proved.

Lemma 3.2. Suppose $|q|<1, k \geqq \max (1,2 \lambda-1)$. Let $H_{\lambda, k, i}^{\#}(x), 0 \leqq i \leqq k$ and $J_{\lambda, k, i}^{\#}(x), 1 \leqq i \leqq k$ be any collection of functions analytic in $x$ in the neighborhood of zero which satisfy

$$
\begin{gathered}
H_{\lambda, k, i}^{\#}(x)-H_{\lambda, k, i-1}^{\#}(x)=\left(x q^{\lambda+1}\right)^{i-1} J_{\lambda, k, k-i+1}^{\#}\left(x q^{\lambda+1}\right), \quad 1 \leqq i \leqq k ; \\
H_{\lambda, k, 0}^{\#}(x)=0 ; \\
J_{\lambda, k, i}^{\#}(x)=\sum_{j=0}^{\min (i, \lambda)} x^{j} \sigma_{j}(\lambda) H_{\lambda, k, i-j}^{\#}(x), \quad 1 \leqq i \leqq k-\lambda+1 ; \\
J_{\lambda, k, k-\lambda+1+j}^{\#}(x)=\sum_{r=0}^{\lambda} x^{r} \sigma_{r}(\lambda) H_{\lambda, k, k-\lambda+1+j-r}^{\#}(x) \\
\quad-\sum_{r=0}^{j} g_{1}(k, \lambda, r, j ; x ; q) H_{\lambda, k, r}^{\#}\left(x q^{\lambda+1}\right), \quad 1 \leqq j<\lambda,
\end{gathered}
$$

where $g_{1}(k, \lambda, r, j ; x ; q)$ is defined in Lemma 2.2.

$$
H_{\lambda, k, i}^{\#}(0)=1, \quad 1 \leqq i \leqq k ; \quad J_{\lambda, k, i}^{\#}(0)=1, \quad 1 \leqq i \leqq k .
$$

Then if we define

$$
\begin{gathered}
H_{\lambda, k, i}^{*}(x)=H_{\lambda, k, i}^{\#}(x), \quad 0 \leqq i \leqq k-\lambda+1, \\
=H_{\lambda, k, i}^{\#}(x)-\sum_{r=1}^{j} H_{\lambda, k, r}^{\#}\left(x q^{\lambda+1}\right) x^{k} q^{(\lambda+1)(k-r)} \sum_{m=0}^{j-r} \sigma_{\lambda-m}(\lambda), \\
i=k-\lambda+1+j, 1 \leqq j<\lambda ; \\
J_{\lambda, k, i}^{*}(x)=x^{-k+i}\left(H_{\lambda, k, k-i+1}^{*}\left(x q^{-\lambda-1}\right)-H_{\lambda, k, k-i}^{*}\left(x q^{-\lambda-1}\right)\right),
\end{gathered}
$$

then $H_{\lambda, k, i}^{*}(x)$ and $J_{\lambda, k, i}^{*}(x)$ fulfill the equations of Lemma 3.1 and for $\lambda \leqq i \leqq k$, $J_{\lambda, k, i}^{*}(x)=J_{\lambda, k, i}^{\#}(x)$.

Proof. Obviously (3.9) and (3.10) are fulfilled from (3.20) and (3.15). Clearly for $\lambda \leqq i \leqq k$,

$$
\begin{aligned}
J_{\lambda, k, i}^{*}(x) & =x^{-k+i}\left(H_{\lambda, k, k-i+1}^{*}\left(x q^{-\lambda-1}\right)-H_{\lambda, k, k-i}^{*}\left(x q^{-\lambda-1}\right)\right) \\
& =x^{-k+1}\left(H_{\lambda, k, k-i+1}^{\#}\left(x q^{-\lambda-1}\right)-H_{\lambda, k, k-i}^{\#}\left(x q^{-\lambda-1}\right)\right) \\
& =J_{\lambda, k, i}^{\#}(x) .
\end{aligned}
$$


Now if $\lambda \leqq i \leqq k-\lambda+1,(3.19)$ and (3.16) show that (3.11) is valid. If $i=k-\lambda+1+j$, $1 \leqq j<\lambda$, then

$$
\begin{aligned}
& J_{\lambda, k, k-\lambda+1+j}^{*}(x)=J_{\lambda, k, k-\lambda+1+j}^{\#}(x) \\
& =\sum_{r=0}^{j-1} x^{r} \sigma_{r}(\lambda) H_{\lambda, k, k-\lambda+1+j-r}^{\#}(x)+\sum_{r=j}^{\lambda} x^{r} \sigma_{r}(\lambda) H_{\lambda, k, k-\lambda+1+j-r}^{\#}(x) \\
& -\sum_{r=0}^{j} g_{1}(k, \lambda, r, j ; x ; q) H_{\lambda, k, r}^{\#}\left(x q^{\lambda+1}\right) \\
& =\sum_{r=0}^{j-1} x^{r} \sigma_{r}(\lambda)\left(H_{\lambda, k, k-\lambda+1+j-r}^{*}(x)+\sum_{v=1}^{j-r} H_{\lambda, k, v}^{*}\left(x q^{\lambda+1}\right)\left(x q^{\lambda+1}\right)^{k} q^{-v(\lambda+1)}\right. \\
& +\sum_{r=j}^{\lambda} x^{r} \sigma_{r}(\lambda) H_{\lambda, k, k-\lambda+1+j-r}^{*}(x) \\
& -\sum_{r=0}^{j} g_{1}(k, \lambda, r, j ; x ; q) H_{\lambda, k, r}^{*}\left(x q^{\lambda+1}\right) \\
& =\sum_{r=0}^{\lambda} x^{r} \sigma_{r}(\lambda) H_{\lambda, k, k-\lambda+1+j-r}^{*}(x) \\
& +\sum_{v=1}^{j} H_{\lambda, k, r}^{*}\left(x q^{\lambda+1}\right) x^{k} q^{(k-v)(\lambda+1)} \sum_{r=0}^{j-v} x^{r} \sigma_{r}(\lambda) \sum_{w=0}^{j-r-v} \sigma_{\lambda-w}(\lambda) \\
& -\sum_{r=0}^{j} g_{1}(k, \lambda, r, j ; x ; q) H_{\lambda, k, r}^{*}\left(x q^{\lambda+1}\right) \\
& =\sum_{r=0}^{\lambda} x^{r} \sigma_{r}(\lambda) H_{\lambda, k, k-\lambda+1+j-r}^{*}(x), \\
& \left.\sum_{w=0}^{j-r-v} \sigma_{\lambda-w}(\lambda)\right)
\end{aligned}
$$

where the last equation follows by Lemma 2.2; thus again we have (3.11).

Finally, if $1 \leqq i<\lambda$, then

$$
\begin{aligned}
J_{\lambda, k, i}^{*}(x)= & x^{-k+i}\left(H_{\lambda, k, k-i+1}^{\#}\left(x q^{-\lambda-1}\right)-\sum_{v=1}^{\lambda-i} H_{\lambda, k, v}^{\#}(x) x^{k} q^{-v(\lambda+1)} \sum_{w=0}^{\lambda-i-v} \sigma_{\lambda-w}(\lambda)\right. \\
& \left.\quad-H_{\lambda, k, k-i}^{\#}\left(x q^{-\lambda-1}\right)+\sum_{v=1}^{\lambda-i-1} H_{\lambda, k, v}^{\#}(x) x^{k} q^{-v(\lambda+1)} \sum_{w=0}^{\lambda-i-1-v} \sigma_{\lambda-w}(\lambda)\right) \\
= & J_{\lambda, k, i}^{\#}(x)-x^{i} \sum_{v=1}^{\lambda-i} H_{\lambda, k, v}^{\#}(x) q^{-v(\lambda+1)} \sigma_{i+v}(\lambda) \\
= & \sum_{j=0}^{i} x^{j} \sigma_{j}(\lambda) H_{\lambda, k, i-j}^{\#}(x)-x^{i} \sum_{v=i+1}^{\lambda} H_{\lambda, k, v-i}^{\#}(x) q^{(i-v)(\lambda+1)} \sigma_{v}(\lambda) \\
= & \sum_{j=0}^{i} x^{j} \sigma_{j}(\lambda) H_{\lambda, k, i-j}^{*}(x)-x^{i} \sum_{v=i+1}^{\lambda} H_{\lambda, k, v-i}^{*}(x) q^{(i-v)(\lambda+1)} \sigma_{v}(\lambda),
\end{aligned}
$$

which again is (3.11). 
Now from (3.19) it is clear that $H_{\lambda, k, i}^{*}(0)=1,1 \leqq i \leqq k$, and since $J_{\lambda, k, i}^{*}(x)$ satisfies (3.11), $J_{\lambda, k, i}^{*}(0)=1,1 \leqq i \leqq k$. The analytic nature of these functions is now obvious from (3.19) and (3.11). Thus we have Lemma 3.2.

COROLlaRY 3.3. The set of functions described in the hypothesis of Lemma 3.2 is unique and exists.

Proof. By Lemma 3.1, the $H_{\lambda, k, i}^{*}(x)$ and $J_{\lambda, k, i}^{*}(x)$ defined in Lemma 3.2 are unique, and thus by (3.19), (3.14), and (3.16) so must $H_{\lambda, k, i}^{\#}(x)$ and $J_{\lambda, k, i}^{\#}(x)$ be. For existence take $H_{\lambda, k, i}^{\#}(x)=H_{\lambda, k, i}(x), 0 \leqq i \leqq k-\lambda+1$,

$$
\begin{aligned}
& H_{\lambda, k, i}^{\#}(x)=H_{\lambda, k, i}(x)+\sum_{r=1}^{j} H_{\lambda, k, r}\left(x q^{\lambda+1}\right) x^{k} q^{(\lambda+1)(k-r)} \sum_{m=0}^{j-r} \sigma_{\lambda-m}(\lambda), \\
& \quad i=k-\lambda+1+j, 1 \leqq j<\lambda, \\
& J_{\lambda, k, i}^{\#}(x)=J_{\lambda, k, i}(x), \quad \lambda \leqq i \leqq k, \\
& J_{\lambda, k, i}^{\#}(x)=\sum_{j=0}^{i} x^{j} \sigma_{j}(\lambda) H_{\lambda, k, i-j}(x), \quad 1 \leqq i<\lambda .
\end{aligned}
$$

Thus we have the corollary.

We are now in a position to establish our main result.

Proof of Theorem 1. Let $P_{\lambda, k, a}(m, n), 1 \leqq a \leqq k$, denote the number of partitions of $n$ into $m$ parts of the form $n=\sum_{i=1}^{\infty} f_{i} \cdot i$, where $f_{i}+\cdots+f_{i+\lambda} \leqq k-1, f_{i(\lambda+1)}+\cdots$ $+f_{(i+1)(\lambda+1)} \leqq k-1, f_{1}+\cdots+f_{\lambda+1} \leqq a-1, f_{b}>1$ then $\lambda+1 \mid b$ (equivalently, $n=$ $b_{1}+\cdots+b_{m}, b_{i} \geqq b_{i+1}, b_{i}-b_{i+k-1} \geqq \lambda+1$ with strict inequality if $\lambda+1 \mid b_{i}$, the total number of parts $\leqq \lambda+1$ does not exceed $a-1$, and only multiples of $\lambda+1$ may be repeated).

Let $Q_{\lambda, k, a}(m, n)$ denote the number of partitions described above in which $f_{1}=f_{2}=\cdots=f_{\lambda}=0$ (i.e., the smallest part is $\geqq \lambda+1$ ).

Let

$$
\begin{array}{ll}
J_{\lambda, k, a}^{\prime}(x)=1+\sum_{m=1}^{\infty} \sum_{n=1}^{\infty} P_{\lambda, k, a}(m, n) x^{m} q^{n}, & 1 \leqq a \leqq k \\
H_{\lambda, k, a}^{\prime}(x)=1+\sum_{m=1}^{\infty} \sum_{n=1}^{\infty} Q_{\lambda, k, a}(m, n) x^{m} q^{n}, & 1 \leqq a \leqq k \\
H_{\lambda, k, 0}^{\prime}(x)=0 .
\end{array}
$$

By comparison with the generating function for the number of ordinary partitions of $n$ into $m$ parts, we see that we have convergence for $|q|<1,|x|<|q|^{-1}$; thus, for given $|q|<1$, the above are analytic in a circle of radius $>1$ around $x=0$.

Also $H_{\lambda, k, a}^{\prime}(0)=J_{\lambda, k, a}^{\prime}(0)=1,1 \leqq a \leqq k$.

Our next object is to show that these functions satisfy (3.14)-(3.17).

Beginning with (3.14), we consider $Q_{\lambda, k, i}(m, n)-Q_{\lambda, k, i-1}(m, n)$; this expression enumerates those partitions considered by $Q_{\lambda, k, i}(m, n)$ in which $\lambda+1$ appears 
exactly $i-1$ times. Let us now subtract $\lambda+1$ from each part in such a partition; the number being partitioned is reduced to $n-m(\lambda+1)$, and the number of parts appearing is now $m-i+1$. Since we had originally

$$
f_{\lambda+2}+\cdots+f_{2 \lambda+2}=k-1-f_{\lambda+1}=(k-i+1)-1,
$$

we have after subtraction

$$
f_{1}+\cdots+f_{\lambda+1}=(k-i+1)-1 .
$$

Otherwise the conditions on the summands are just shifted but unaltered. Thus we are now considering a partition of the type enumerated by

$$
P_{\lambda, k, k-i+1}(m-i+1, n-m(\lambda+1)) \text {. }
$$

The above procedure establishes a one-to-one correspondence between the partitions enumerated by $Q_{\lambda, k, i}(m, n)-Q_{\lambda, k, i-1}(m, n)$ and those enumerated by $P_{\lambda, k, k-i+1}(m-i+1, n-m(\lambda+1))$. Hence

$$
Q_{\lambda, k, i}(m, n)-Q_{\lambda, k, i-1}(m, n)=P_{\lambda, k, k-i+1}(m-i+1, n-m(\lambda+1)) .
$$

In terms of generating functions, this implies

$$
H_{\lambda, k, i}^{\prime}(x)-H_{\lambda, k, i-1}^{\prime}(x)=\left(x q^{\lambda+1}\right)^{i-1} J_{\lambda, k, k-i+1}^{\prime}\left(x q^{\lambda+1}\right),
$$

which is (3.14).

(3.15) is by definition.

We now treat (3.16). I claim that for $1 \leqq i \leqq k-\lambda+1$

$$
x^{j} \sigma_{j}(\lambda) H_{\lambda, k, i-j}^{\prime}(x), \quad 0 \leqq j \leqq \min (i, \lambda),
$$

is the generating function for the number of partitions of the type enumerated by $P_{\lambda, k, i}(m, n)$ in which exactly $j$ parts $\leqq \lambda$ appear.

By Lemma 2.3, (3.21) is the generating function for partitions in which there are $j$ distinct parts $\leqq \lambda$ and the parts $\geqq \lambda+1$ satisfy the desired conditions. Is it possible then that any of the conditions on the partitions enumerated by $P_{\lambda, k, i}(m, n)$ could be violated? This is not possible because for $1 \leqq v \leqq \lambda$

$$
f_{v}+\cdots+f_{\lambda} \leqq j ; \quad f_{\lambda+1} \leqq i-j-1 ; \quad f_{\lambda+2}+\cdots+f_{\lambda+v} \leqq v-1 ;
$$

hence

$$
f_{v}+\cdots+f_{v+\lambda} \leqq j+i-j-1+v-1=i+v-2 .
$$

If $v=1$, this is $f_{1}+\cdots+f_{\lambda+1} \leqq i-1$ as desired, and if $1<v \leqq \lambda$, this is $f_{v}+\cdots$ $+f_{v+\lambda} \leqq i+v-2 \leqq k-\lambda+1+\lambda-2=k-1$ as desired.

Conversely it is easily seen that any partition of the type enumerated by $P_{\lambda, k, i}(m, n)$ with exactly $j$ parts $\leqq \lambda$ is of the type considered by (3.21). 
Thus clearly

$$
\begin{aligned}
J_{\lambda, k, i}^{\prime}(x) & =\sum_{j=0}^{i} x^{j} \sigma_{j}(\lambda) H_{\lambda, k, i-j}^{\prime}(x) \\
& =\sum_{j=0}^{\min (i, \lambda)} x^{j} \sigma_{j}(\lambda) H_{\lambda, k, i-j}^{\prime}(x), \quad 1 \leqq i \leqq k-\lambda+1,
\end{aligned}
$$

which is (3.16).

For (3.17) we note that the above procedure must be modified since now in considering (3.21) with $i>k-\lambda+1$ it is indeed possible that there exists $v$ with $1<v \leqq \lambda$, such that $f_{v}+\cdots+f_{v+\lambda} \geqq k$.

Indeed by the preceding argument for $1 \leqq j<\lambda$

$$
\sum_{r=0}^{\lambda} x^{r} \sigma_{r}(\lambda) H_{\lambda, k, k-\lambda+1+j-r}^{\prime}(x)-J_{\lambda, k, k-\lambda+1+j}^{\prime}(x)
$$

is the generating function for partitions of the type enumerated by

$$
P_{\lambda, k, k-\lambda+1+j}(m, n)
$$

except for the fact that for some $v, 1<v \leqq \lambda, f_{v}+\cdots+f_{v+\lambda} \geqq k$.

On the other hand, let us consider

$$
g_{1}(k, \lambda, r, j ; x ; q) H_{\lambda, k, r}^{\prime}\left(x q^{\lambda+1}\right), \quad 0 \leqq r<\lambda .
$$

I claim that this is the generating function for partitions of the type described in the preceding paragraph in which $f_{\lambda+1}+\cdots+f_{2 \lambda+1}=k-r$.

By Lemma 2.3, Lemma 2.2 (and the fact that $H_{\lambda, k, r}^{\prime}\left(x q^{\lambda+1}\right)$ is the generating function for partitions of the type enumerated by $P_{\lambda, k, r}(m, n)$ with the added restriction that $\left.f_{1}=f_{2}=\cdots=f_{2 \lambda+1}=0, f_{2 \lambda+2} \leqq r-1\right)$, we see that (3.23) is the generating function for partitions of $n$ into $m$ parts of the form $n=\sum_{i=1}^{\infty} f_{i} \cdot i$, where

$$
\begin{aligned}
f_{1}+\cdots+f_{\lambda+1} & \leqq k-\lambda+j ; \\
f_{\lambda+1}+\cdots+f_{2 \lambda+1} & =k-r ; \\
f_{v}+\cdots+f_{v+\lambda} & \geqq k \quad \text { for some } v, \quad 1<v \leqq \lambda ; \\
f_{a} & >1 \quad \text { implies } \lambda+1 \mid a ; \\
f_{m}+\cdots+f_{m+\lambda} & \leqq k-1, \quad \text { for } m \geqq 2 \lambda+2 ; \\
f_{m(\lambda+1)}+\cdots+f_{(m+1)(\lambda+1)} & \leqq k-1, \quad m \geqq 2 ; \\
f_{2 \lambda+2} & \leqq r-1 .
\end{aligned}
$$

We shall establish the claim made for (3.23) if we can show that the following two conditions hold for the partitions considered.

$$
\begin{aligned}
f_{m}+\cdots+f_{m+\lambda} & \leqq k-1, \text { for } \lambda+1<m<2 \lambda+2 \\
f_{\lambda+1}+\cdots+f_{2 \lambda+2} & \leqq k-1 .
\end{aligned}
$$


Now (3.32) is clear from (3.25) and (3.30). As for (3.31) we have

$$
f_{m}+\cdots+f_{2 \lambda+1}+f_{2 \lambda+3}+\cdots+f_{m+\lambda} \leqq \lambda ; \quad f_{2 \lambda+2} \leqq r-1 ;
$$

therefore

$$
f_{m}+\cdots+f_{m+\lambda} \leqq \lambda+r-1 \leqq \lambda+\lambda-2=2 \lambda-2 \leqq k-1 .
$$

Thus (3.23) is the desired generating function.

Consequently for $1 \leqq j<\lambda$,

$$
\begin{aligned}
\sum_{r=0}^{\lambda} x^{r} \sigma_{r}(\lambda) H_{\lambda, k, k-\lambda+1+j-r}^{\prime}( & (x)-J_{\lambda, k, k-\lambda+1+j}^{\prime}(x) \\
= & \sum_{r=0}^{\lambda-1} g_{1}(k, \lambda, r, j ; x ; q) H_{\lambda, k, r}^{\prime}\left(x q^{\lambda+1}\right) \\
= & \sum_{r=0}^{j} g_{1}(k, \lambda, r, j ; x ; q) H_{\lambda, k, r}^{\prime}\left(x q^{\lambda+1}\right),
\end{aligned}
$$

which is (3.17); the second to last equation is summed to $\lambda-1$ only because (3.25) and (3.24) contradict (3.26) if $r \geqq \lambda$.

Thus by Corollary 3.3 and its proof,

$$
J_{\lambda, k, i}^{\prime}(x)=J_{\lambda, k, i}(x), \quad \lambda \leqq i \leqq k .
$$

Finally

$$
\begin{aligned}
& 1+\sum_{n=1}^{\infty} B_{\lambda, k, i}(n) q^{n} \\
& =1+\sum_{n=1}^{\infty} \sum_{m=1}^{\infty} P_{\lambda, k, i}(m, n) q^{n} \\
& =J_{\lambda, k, i}(1) \\
& =\prod_{j=1}^{\infty}\left(1+q^{j}\right)\left(1+q^{j(\lambda+1)}\right)^{-1}\left(1-q^{j(\lambda+1)}\right)^{-1}\left(1-q^{(2 k-\lambda+1)(\lambda+1) j-\left(i-\frac{1}{2} \lambda\right)(\lambda+1)}\right) \\
& \cdot\left(1-q^{(2 k-\lambda+1)(\lambda+1)(j-1)+\left(i-\frac{1}{2} \lambda\right)(\lambda+1)}\right)\left(1-q^{(2 k-\lambda+1)(\lambda+1) j}\right)
\end{aligned}
$$

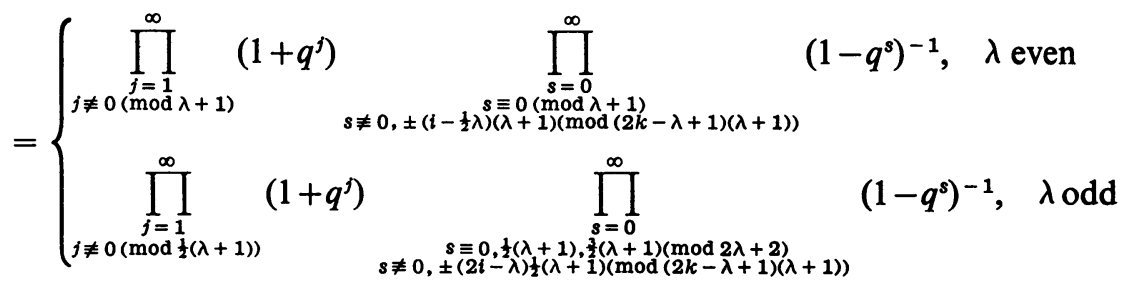

$$
\begin{aligned}
& =1+\sum_{n=1}^{\infty} A_{\lambda, k, i}(n) q^{n}
\end{aligned}
$$

where the third to last equation follows from [3, equation (2.9)]. Hence $A_{\lambda, k, i}(n)$ $=B_{\lambda, k, i}(n)$ and Theorem 1 is established. 
4. Extension of Theorem 1. We extend our definition of $B_{\lambda, k, a}(n)$ as follows. Let $B_{\lambda, k, a}^{*}(n)$ denote those partitions of $n$ enumerated by $B_{\lambda, k, a}(n)$ such that for any integer $j$ in the interval $\left[1, \frac{1}{2}(\lambda+1)\right]$, there must be at most $a-j$ parts of the partition in the interval $[j, \lambda-j+1]$ (in terms of frequency notation the new condition is $\left.f_{j}+\cdots+f_{\lambda-j+1} \leqq a-j, 1 \leqq j \leqq \frac{1}{2}(\lambda+1)\right)$. We define $P_{\lambda, k, a}^{*}(m, n)$ similarly.

We remark that for $a \geqq \lambda, B_{\lambda, k, a}^{*}(n)=B_{\lambda, k, a}(n)$. This is because the new conditions are automatically fulfilled for $a \geqq \lambda$, since the number of summands in the interval $[j, \lambda-j+1]$ is $\leqq \min (a-1, \lambda-2 j+2) \leqq \min (a-1, a-2 j+2) \leqq a-j$.

In Theorem 1 we note that $A_{\lambda, k, a}(n)$ is well defined for $\frac{1}{2} \lambda<a \leqq k$. We now have the following extension of Theorem 1 .

THEOREM 2. $A_{\lambda, k, a}(n)=B_{\lambda, k, a}^{*}(n)$ for $\frac{1}{2} \lambda<a \leqq k, k \geqq 2 \lambda-1$.

This theorem will be easy to deduce from Theorem 1 after we digress briefly for a study of a new auxiliary partition function.

Let $\varphi(i, j, \lambda ; n), \frac{1}{2} \lambda<i \leqq \lambda, i \leqq j \leqq \lambda$, be the number of partitions of $n$ into $2 i-j$ distinct parts of the form $n=\sum_{e=1}^{\lambda} f_{e} \cdot e$ where at least one of the following inequalities holds

$$
f_{b}+\cdots+f_{\lambda-b+1}>i-b, \quad 1 \leqq b \leqq \frac{1}{2}(\lambda+1) .
$$

Let $\psi(i, j, \lambda ; q)=\sum_{n=0}^{\infty} \varphi(i, j, \lambda ; n) q^{n}$.

LEMMA 4.1. For $\frac{1}{2} \lambda<i \leqq \lambda, i \leqq j \leqq \lambda$,

$$
\psi(i, j, \lambda ; q)=q^{(i-j)(\lambda+1)} \sigma_{j}(\lambda) .
$$

Proof. Now if $i=j$, then $2 i-j=i$. Therefore $f_{1}+\cdots+f_{\lambda}=i>i-1$. Hence $\psi(i, i, \lambda ; q)$ is just the generating function for partitions into $i$ distinct parts all $\leqq \lambda$. Thus

$$
\psi(i, i, \lambda ; q)=\sigma_{i}(\lambda)
$$

We now wish to show

$$
\psi(i+1, j, \lambda ; q)=q^{\lambda+1} \psi(i, j, \lambda ; q) ;
$$

(4.4) together with (4.3) imply (4.2) by mathematical induction.

We proceed as follows. For a given partition, $\pi$, of the type enumerated by $\varphi(i, j, \lambda ; n)$, define

$$
\begin{aligned}
& m(\pi)=\max _{1 \leqq b \leqq \frac{1}{2}(\lambda+1)} f_{b}+\cdots+f_{\lambda-b+1}-i+b ; \\
& U(\pi)=\min \left\{b \mid f_{b}+\cdots+f_{\lambda-b+1}-i+b=m(\pi)\right\} \\
& L(\pi)=\max \left\{b \mid f_{b}+\cdots+f_{\lambda-b+1}-i+b=m(\pi)\right\} .
\end{aligned}
$$

We now note that

$$
f_{U(\pi)-1}=f_{\lambda-U(\pi)+2}=0 ; \quad f_{L(\pi)}=f_{\lambda-L(\pi)+1}=1 ;
$$


(4.8) is correct because the falsity of the first assertion would contradict the minimality of $U(\pi)$, and the falsity of the second assertion would contradict the maximality of $L(\pi)$ (note that $U(\pi)>1$ for $j>i$ because $i-1 \geqq 2 i-j=f_{1}+\cdots+f_{\lambda}$ in contrast with the fact that $(4.1)_{\mathrm{b}}$ is valid for some $\left.b\right)$.

We now prove (4.4). If $\pi$ is a partition enumerated by $\varphi(i, j, \lambda ; n)$ delete from $\pi$ the summands $L(\pi)$ and $\lambda-L(\pi)+1$, and call the new partition $\pi^{\prime} . \pi^{\prime}$ is a partition of $n-\lambda-1$ with $2(i-1)-j$ parts.

$$
U\left(\pi^{\prime}\right)=L(\pi)+1 ;
$$

this is because if $b<L(\pi)+1$, then in $\pi^{\prime}$ we have

$$
f_{b}^{\prime}+\cdots+f_{\lambda-b+1}^{\prime}-(i-1)+b=f_{b}+\cdots+f_{\lambda-b+1}-2-i+1+b \leqq m(\pi)-1,
$$

while for $b \geqq L(\pi)+1$

and

$$
f_{b}^{\prime}+\cdots+f_{\lambda-b+1}^{\prime}-(i-1)+b=f_{b}+\cdots+f_{\lambda-b+1}-i+1+b \leqq m(\pi),
$$

$$
\begin{aligned}
f_{L(\pi)+1}^{\prime}+\cdots+f_{\lambda-L(\pi)}^{\prime}-(i-1)+ & L(\pi)+1 \\
& =f_{L(\pi)}+\cdots+f_{\lambda-L(\pi)+1}-2-i+1+L(\pi)+1 \\
& =m(\pi) .
\end{aligned}
$$

Now since originally $m(\pi)>0$, we see that $m(\pi)=m\left(\pi^{\prime}\right)>0$, and therefore $\pi^{\prime}$ is a partition of the type enumerated by $\varphi(i-1, j, \lambda ; n-\lambda-1)$.

Conversely if $\pi^{\prime}$ is a partition of the type enumerated by $\varphi(i-1, j, \lambda ; n-\lambda-1)$ then introduce the summands $U\left(\pi^{\prime}\right)-1$, and $\lambda-U\left(\pi^{\prime}\right)+2$ and call the new partition $\pi^{\prime \prime} . \pi^{\prime \prime}$ is a partition of $n$ into $2 i-j$ parts.

$$
L\left(\pi^{\prime \prime}\right)=U\left(\pi^{\prime}\right)-1 ;
$$

this is because if $b>U\left(\pi^{\prime}\right)-1$

while for $b \leqq U\left(\pi^{\prime}\right)-1$

$$
\begin{aligned}
f_{b}^{\prime \prime}+\cdots+f_{\lambda-b+1}^{\prime \prime}-i+b & =f_{b}^{\prime}+\cdots+f_{\lambda-b+1}^{\prime}-(i-1)+b-1 \\
& \leqq m\left(\pi^{\prime}\right)-1,
\end{aligned}
$$

$$
\begin{aligned}
f_{b}^{\prime \prime}+\cdots+f_{\lambda-b+1}^{\prime \prime}-i+b & =f_{b}^{\prime}+\cdots+f_{\lambda-b+1}^{\prime}-(i-1)+b+2-1 \\
& \leqq m\left(\pi^{\prime}\right)-1+2-1=m\left(\pi^{\prime}\right),
\end{aligned}
$$

and

$$
\begin{aligned}
f_{U\left(\pi^{\prime}\right)-1}^{\prime \prime}+\cdots+f_{\lambda-U\left(\pi^{\prime}\right)+2}^{\prime \prime} & i+U\left(\pi^{\prime}\right)-1 \\
= & f_{U\left(\pi^{\prime}\right)}^{\prime}+\cdots+f_{\lambda-U\left(\pi^{\prime}\right)+1}^{\prime}+2-(i-1)+U\left(\pi^{\prime}\right)-2 \\
= & m\left(\pi^{\prime}\right) .
\end{aligned}
$$

Thus we see that the above procedure establishes a one-to-one correspondence between the partitions enumerated by $\varphi(i, j, \lambda ; n)$ and those enumerated by $\varphi(i-1, j, \lambda ; n-\lambda-1)$. Therefore

$$
\varphi(i+1, j, \lambda ; n)=\varphi(i, j, \lambda ; n-\lambda-1) .
$$

We deduce (4.4) immediately from (4.11). Thus we have established Lemma 4.1. 
Proof of Theorem 2. We have already noted that Theorem 2 is just Theorem 1 if $a \geqq \lambda$. We thus need only treat $\frac{1}{2} \lambda<a<\lambda$. Under these conditions we let $J_{\lambda, k, a}^{\prime \prime}(x)$ be the generating function for $P_{\lambda, k, a}^{*}(m, n)$. Then as in the proof of Theorem 1,

$$
\begin{aligned}
J_{\lambda, k, a}^{\prime \prime}(x) & =J_{\lambda, k, a}^{\prime}(x)-\sum_{j=a}^{\lambda} x^{2 a-j} \psi(a, j, \lambda ; q) H_{\lambda, k, j-a}(x) \\
& =\sum_{j=0}^{a} x^{j} \sigma_{j}(\lambda) H_{\lambda, k, a-j}(x)-\sum_{j=a+1}^{\lambda} x^{2 a-j} \psi(a, j, \lambda ; q) H_{\lambda, k, j-a}(x)
\end{aligned}
$$

for the first term on the right-hand side (from the proof of Theorem 1) generates the partitions enumerated by $P_{\lambda, k, a}(m, n)$ and the second term, by the definition of $\psi$ and Lemma 2.3, just removes those partitions enumerated by $P_{\lambda, k, a}(m, n)$ which are not enumerated by $P_{\lambda, k, a}^{*}(m, n)$.

Now on the other hand from (3.6) and (3.7)

$$
J_{\lambda, k, a}(x)=\sum_{j=0}^{a} x^{j} \sigma_{j}(\lambda) H_{\lambda, k, a-j}(x)-x^{a} \sum_{j=a+1}^{\lambda} q^{(a-j)(\lambda+1)} \sigma_{j}(\lambda) H_{\lambda, k, j-a}(x) .
$$

Consequently by Lemma 4.1

$$
J_{\lambda, k, a}^{\prime \prime}(1)=J_{\lambda, k, a}(1) .
$$

The remainder of the proof is now identical with that of Theorem 1 .

5. Conclusion. Let us define $K(\lambda)$ to be that integer such that for $k \geqq K(\lambda)$ Theorem 2 (and consequently Theorem 1 ) holds.

ConjeCture. $K(\lambda)=\lambda$.

It is fairly easy to show that $K(2)=2$, and indeed numerical evidence indicates the truth of $K(\lambda)=\lambda$ for $\lambda=3,4,5$. The main problem in proving the conjecture lies in the fact that the $q$-difference equations become much more involved for smaller values of $k$ and the related partition identities become even more cumbersome.

A further interesting question seems to be whether or not even smaller values of $k$ may be taken provided new restrictions are put on the partitions enumerated by $B_{\lambda, k, a}(n)$. As an example, Schur has proved $[6$, p. 495] that

$$
A_{3,2,2}(n)=B_{3,2,2}^{0}(n)
$$

where $B_{3,2,2}^{0}(n)$ denotes the number of partitions enumerated by $B_{3,2,2}(n)$ with the added restriction that no parts are $\equiv 2(\bmod 4)$.

\section{REFERENCES}

1. H. L. Alder, The nonexistence of certain identities in the theory of partitions and compositions, Bull. Amer. Math. Soc. 54 (1948), 712-722.

2. G. E. Andrews, A generalization of the Göllnitz-Gordon partition theorems, Proc. Amer. Math. Soc. 18 (1967), 945-952. 
3. G. E. Andrews, q-difference equations for certain well-poised basic hypergeometric series, Quart. J. Math. Oxford Ser. (2) 19 (1968), 433-447.

4. B. Gordon, A combinatorial generalization of the Rogers-Ramanujan identities, Amer. J. Math. 83 (1961), 393-399.

5. G. H. Hardy and E. M. Wright, An introduction to the theory of numbers, 4th ed., Oxford Univ. Press, Oxford, 1960.

6. I. J. Schur, Zur additiven Zahlentheorie, S.-B. Deutsch. Akad. Wiss. Berlin Math.-Mat. Kl. (1926), 488-495.

The Pennsylvania State University,

University Park, Pennsylvania 